\title{
The Savvy Survey \#9: Gaining Institutional Review Board Approval for Surveys ${ }^{1}$
}

\author{
Colleen E. Gariton and Glenn D. Israel ${ }^{2}$
}

\section{Introduction}

What is the IRB? Do I need to get IRB approval? Will this take a lot of time on my part? What counts as informed consent? Getting IRB approval seems like a daunting task to many people in academia and Extension, but as long as you are not doing research that has the potential to harm or impact your subjects, it does not have to be. This publication will discuss what the IRB is, when and how to get IRB approval for a survey, getting informed consent (which is good to get even if you do not need IRB approval), and where to get help for Extension professionals wanting to perform research or publish findings from a survey.

\section{What is the IRB?}

The IRB is the Institutional Review Board, which is there to ensure that research involving a human subject being conducted by an institution or university is ethical and legal. (If you happen to be involved in research on animal subjects, then you need approval from the Institutional Animal Care and Use Committee [IACUC], and you need to consult other resources.) For all human research, from the most invasive (e.g., medical implants) to the least (e.g., an anonymous five-question survey), you need IRB approval. The IRB is important to make sure that human subjects are not being exploited and will not be at excessive risk of harm. At UF, there are two IRBs you might need to work with: IRB-01, the medical IRB (very rare for
Extension), and IRB-02, for social science studies and most of Extension.

\section{Do I need IRB approval/what counts as research?}

Research involves "a systematic investigation (including research development, testing and evaluation) designed to develop or contribute to generalizable knowledge" (UF Institutional Review Board, 2018, "What is Research?" para. 1). Survey results are considered to be research when you use the information to make a presentation for a conference, publish a journal article or proceedings, or document findings in a report because these activities are "part and parcel" with creating generalizable knowledge. On the other hand, surveys conducted in support of sound programming practice, such as collecting data for a needs assessment, marketing of the program, program improvement, or accountability reporting, are not considered research and do not need IRB approval as long as the information is not used in ways that develop generalizable knowledge (Harder, n.d.). If you are working with and collecting data from vulnerable populations (e.g., children/youth, the mentally ill, prisoners, pregnant women), you should get IRB approval because they are considered most in need of protection. If you are planning to collect data from $4-\mathrm{H}$ youth, check with the UF/IFAS Extension 4-H Youth Development program to learn what measures already have IRB approval.

1. This document is AEC730, one of a series of the Department of Agricultural Education and Communication, UF/IFAS Extension. Original publication date June 2021. Visit the EDIS website at https://edis.ifas.ufl.edu for the currently supported version of this publication.

2. Colleen E. Gariton, graduate student; and Glenn D. Israel, professor; Department of Agricultural Education and Communication, UF/IFAS Extension, Gainesville, FL 32611.

The Institute of Food and Agricultural Sciences (IFAS) is an Equal Opportunity Institution authorized to provide research, educational information and other services

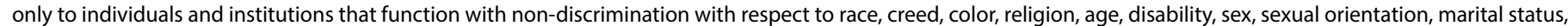

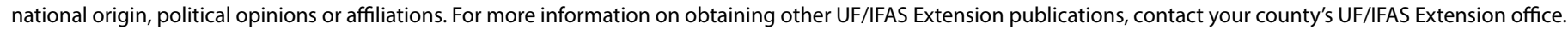
U.S. Department of Agriculture, UF/IFAS Extension Service, University of Florida, IFAS, Florida A \& M University Cooperative Extension Program, and Boards of County Commissioners Cooperating. Nick T. Place, dean for UF/IFAS Extension. 


\section{Why is approval from the IRB important?}

Submitting your work for IRB approval gives your research a review by an outside committee that may be able to see concerns with how the survey communicates and engages with potential participants that might not be as apparent to people conducting the study. Their approval maintains that the survey procedures work to minimize risk to participants and to ensure that risks that may occur are reasonable in relation to anticipated benefits; to create equitable selection (the proposed research does not discriminate among individuals in the population in being selected or not); to protect vulnerable individuals; to provide informed consent and the right to withdraw; to monitor data for adverse effects; and to ensure the privacy and confidentiality of participants.

\section{What do I need to do to get IRB approval?}

1. Set up the VPN client on your computer. The UF IRB website requires the VPN. Visit https://it.ufl.edu/ict/ documentation/network-infrastructure/vpn/ for instructions and a link to download the client.

2. Next, take the IRB training through the UF training system. Make sure to do this through UF, not UF Health. Access the training at https://mytraining-ufshands. sumtotal.host/core/pillarRedirect? relyingParty=LM\&url =app\%2Fmanagement\%2FLMS_ActDetails.aspx\%3FActi vityId\%3D44218\%26UserMode\%3D0. This training takes about 20-45 minutes to complete and is good for 3 years. While formerly there were several IRB trainings at UF, now there is only one. Note that training can take up to 3 days to show on the IRB website as completed, and you cannot enter information for a new study beforehand.

3. Create your research protocol, questions/survey, informed consent, and any other material needed. Make sure these all meet the criteria for protecting participants, listed in the previous section.

4. Register for IRB at http://irb.ufl.edu/myirb/registration3rd.html. You must log into the VPN before you can enter and complete the registration process. When registering you will have to provide your name, address, phone number, title, type of staff role, and department/ organization. Once completed, you will receive one email confirming your registration and another when you can add a new study. This usually takes about an hour but may take as long as a day or two.

5. After receiving notice about being registered, you can create a new study. Again, you must log into the VPN and then log into MyIRB at https://my.irb.ufl.edu/UFLIRB. Here you will provide all the information about your study. This information can take some time to enter, and the software can take some time to move through. Some helpful tips are listed below.

6. To see your study's progress after it is submitted, check the IRB studies tab on the website. You may be told to revise something, which you can complete and submit on the revise tab of this page. When your study is approved, it will update here.

7. Start your survey, data collection, analysis, and writing for publication. Also make sure to report any adverse events or changes in the protocol through the revision tab on the IRB site.

8. If you have already collected data from participants and then decide you want to use this in research, you may be able to, but it is a little more difficult, and you must apply for IRB approval post hoc. Contact the IRB office to make sure you have everything you need.

\section{Proposal Submission Tips}

First, you need to determine which IRB should review your submission; IRB-02 is the appropriate choice for most Extension studies. You must also answer whether the survey is multi-institution, which means whether it involves an organization outside of UF. Then you need to know what type of research is exempt. Research is most often exempt for Extension if you are not dealing with a vulnerable population (youth, prisoners, the mentally ill, or pregnant women) or sensitive subjects (illegal or criminal behaviors, abuse, health-related information covered by HIPAA, etc.). If you are dealing with one of these, select expedited as the type. You may also need to select expedited if you have access to participants' identification and answers. If you select the wrong category, your answers to subsequent questions will be flagged as errors, the site will respond that your study cannot be exempt, and then you must change the category in order to complete the submission process. You will also need to list funding if applicable, but often this is not needed for most Extension programs.

If you have coinvestigators or others involved in the study, they must agree to participate by logging into the IRB site 
too. Make sure to let them know, send them reminder emails, and help them get registered if they have not already done so.

To save time or if you are a first-time submitter, you can use the examples or protocols given on the IRB's site. All you have to do is fill in your information for your research into these documents.

\section{Informed Consent and Assent}

Informed consent is one of the most important parts of developing a survey's protocol. This should not just be with studies that require IRB approval, but anytime you collect information from participants. Informed consent tells potential participants about the purpose of the study, what will happen during the study, how they will be involved, what will happen with the data after you receive it, whether the information is confidential, their right to refuse to continue or to leave at any time, and how and where they can get the information from the study once it is completed. This information needs to be communicated before a person starts a survey or interview.

Youth under the age of 18 are considered a vulnerable population, and you will need parent/guardian informed consent. This must be done prior to collecting data. There are three options for parent informed consent: the first is to ask them to sign something and return it, either electronically or on paper; the second is having consent as an enrollment process of $4-\mathrm{H}$ (if they do not consent at enrollment, then they never receive an evaluation of the program); and the third is passive consent. With passive consent, you send an email or paper to the guardian stating that if they do not respond that they do not want their child involved before the data is to be collected, then their child will be included. In every case, you must provide your contact information. Each option has its benefits; for advice, you can always contact state $4-\mathrm{H}$ staff. You should also provide the youth with informed consent at the beginning of the survey or interview because they have the right to not participate. Their agreeing to take part is called assent.

\section{Incorporating Informed Consent in Your Study or Interview}

When you are conducting an electronic survey, you can send the informed consent as part of the email to invite participants, but it is better to have it be the first page of the survey they see (i.e., on the landing page). On the landing page, you can ask an individual to click "yes to agree" to give consent, or you can say by continuing the survey they give consent. When you are conducting a paper survey, you can include the informed consent information in the cover letter. This works for paper surveys sent by mail or given in person. If you are conducting the survey by phone, in person, as a group, or as an interview, you should have a script with the information and then ask if they agree to participate. If required by the IRB, you also might need to have a handout form to give to participants or mail to them to record their consent.

\section{How long does IRB approval take?}

After submission of an exempt study, it usually takes a week or less to be approved or to receive comments for revision if needed. Often the requested revision is related to informed consent. A response for an expedited study may take a couple of weeks, but it is usually fairly fast.

\section{Where can I get help?}

- State specialists, evaluation experts, and UF faculty.

- IRB site: http://irb.ufl.edu/

- IT site for help with VPN: https://helpdesk.ufl. edu/ and https://my.it.ufl.edu/CherwellPortal/ UFITServicePortal?_=112d46d6\#0

\section{Resources}

\section{Information}

http://irb.ufl.edu/irb02/forms-templates-guidelines/irbrev. html

https://www.fda.gov/regulatory-

information/search-fda-guidance-documents/

institutional-review-boards-frequently-asked-questions

\section{Templates}

http://irb.ufl.edu/irb01/forms.html

http://www.bumc.bu.edu/irb/inspir-ii/irb-templates/

http://irb.ufl.edu/irb02/informed-consent-instructionsprocedures/samples.html

http://irb.ufl.edu/irb01/forms/forms-2-2.html 


\section{References}

Harder, A. (n.d.). Guidelines for determining the differences between research and program evaluation. UF/IFAS Extension. https://pdec.ifas.ufl.edu/evaluation/PDEC_EvaluationFactSheet01.pdf

UF Institutional Review Board. (2018). Definition of a human subject research. http://irb.ufl.edu/index/humanrsch. html

\section{Acknowledgment}

We would like to thank Sarah Hensley for her helpful comments on an earlier version of this publication.

\section{Abstract}

Getting IRB approval for your survey can seem like a daunting task to many people in academia and Extension. This publication discusses what the IRB is, when and how to get IRB approval for a survey, getting informed consent (which is good to get even if you do not need IRB approval), and where to get help for Extension professionals wanting to perform research or publish findings from surveys they have completed. Most surveys that receive an IRB review are in the exempt category and can obtain approval to proceed in one or two weeks. Understanding your responsibilities for conducting a survey in an ethical manner is important and expected. 